International Journal of Physical Research, $7(2)(2019) 100-105$
International Journal of Physical Research
SPC
Website: www.sciencepubco.com/index.php/IJPR
Research paper

\title{
An approximate solution of the Yang - Mills equation on a spatially flat FRW cosmological background
}

\author{
V. K. Shchigolev ${ }^{1 *}$ \\ ${ }^{1}$ Department of Theoretical Physics, Ulyanovsk State University, Ulyanovsk, 432000, Russia \\ ${ }^{*}$ Corresponding author E-mail:vkshch@yahoo.com
}

\begin{abstract}
In this paper, an approximate solution for the Yang - Mills equation in a spatially flat Friedmann-Robertson-Walker universe is obtained. For this purpose, the well known method of solution of non-linear differential equations is used, viz. the homotopy perturbations method. This method has been developed as effective technique for solving different non-linear problems. Here, this method allowed us to obtain approximate solution for the essentially non-linear equation for the $\mathrm{SO}_{3}$ Yang-Mills fields on the curved space-time background of the spatially flat Friedmann-Robertson-Walker universe.
\end{abstract}

Keywords: Friedmann-Robertson-Walker Universe, Homotopy Perturbation Method, Yang-Mills Equation.

\section{Introduction}

Numerous attempts to theoretically explain the discovery made twenty years ago, namely the modern accelerated expansion of our Universe [1]-[3] are well known from the literature (see, for example, [4, 5] and references therein). An abundance of explanations of this phenomenon is associated both with various modifications of sources of gravity (dark energy), and with modifications of the theory of gravity itself (see, for example, [6] and references therein). Some dark energy models are based on the conjecture that a vector field can play the role of the dark energy source. The Yang-Mills fields [7] were also considered as one of the possible sources of gravity in such an aspect [8] including the works of the author (see, for example, $[9,10,11,12]$ ). One of the hardly noticeable but curious fact in our study is that our exact solutions for the Yang-Mills fields represent non-zero energy densities and pressures of the Yang-Mills field in the spatially curved Friedmann-Robertson-Walker (FRW) universe (when $\mathrm{k}=+1,-1$ ). At the same time, this solution, being non-zero, yields a zero energy density of the Yang-Mills field in the flat case $(k=0)$.

It would be interesting to find out if there are any solutions to the Yang-Mills equation providing non-zero energy densities in the flat case, even if this field is considered only on the gravity background of the flat FRW universe. In the present work, we obtain such a solution using an approximate method for solving nonlinear equations, namely He's Homotopy Perturbation Method (HPM) [13, 14, 15]. Recently we applied this method for analytical computations in the field of cosmology and astrophysics (see, e.g. [16] - [19]. It can be also mentioned that applications of HPM can be found in the field of astrophysics in different contexts that creates a new research field [20, 21, 22].

In order to apply HPM to the problem of solving the Yang-Mills equation on the gravity background of the flat FRW universe, we use the Wu-Yang ansatz, and reduce the Yang-Mills system of equations in the flat FRW universe to a single radial equation for the radial function. After a certain type of replacement of the radial variable, we reduce the radial equation to a form suitable for solving by the HPM. Applying HPM, we find approximate solution for the components of the Yang-Mills field and graphically illustrate the non-zero character of the components of the energy-momentum tensor of this field in the flat FRW universe.

\section{The Yang-Mills fields in FRW cosmology}

As it is well known, the Lagrangian density of $\mathrm{SO}_{3}$ YM field [7] is as follows

$L_{Y M}=-\frac{1}{16 \pi} F_{i k}^{a} F^{a i k}$

where

$F_{i k}^{a}=\partial_{i} W_{k}^{a}-\partial_{k} W_{i}^{a}+g \varepsilon_{a b c} W_{i}^{b} W_{k}^{c}$ 
is the stress tensor of YM fields $W_{i}^{a}$.

The variation of Lagrangian (1) with respect to YM fields $W_{i}^{a}$ yields the following YM equation

$D_{i}\left(\sqrt{-g} F^{a i k}\right) \equiv \partial_{i}\left(\sqrt{-g} F^{a i k}\right)+\sqrt{-g} \varepsilon^{a b c} W_{i}^{b} F^{c i k}=0$,

where $D_{i}$ stands for the covariant derivative.

The energy-momentum tensor (EMT) of matter can be derived from the Lagrangian of matter. Therefore, the EMT of the YM fields is followed from the variation of YM Lagrangian (1) with respect to the metric of space-time. One can readily obtain the following EMT of YM fields

$T_{i}^{k}=-\frac{1}{4 \pi} F_{i j}^{a} F^{a k j}+\frac{1}{16 \pi} \delta_{i}^{k} F_{m n}^{a} F^{a m n}$.

The Friedmann-Robertson-Walker (FRW) line element can be represented as

$d s^{2}=d t^{2}-a^{2}(t)\left[d r^{2}+\xi^{2}(r) d \Omega^{2}\right]$,

where $a(t)$ is a scale factor of the Universe, and

$\xi_{(r)}=\left\{\begin{array}{cl}\sin (r), & k=+1, \\ r, & k=0, \\ \sinh (r), & k=-1 .\end{array}\right.$

in accordance with the sign of the spatial curvature $k$.

The generalized Wu-Yang ansatz for the $\mathrm{SO}_{3} \mathrm{YM}$ fields can be written down as [11]

$W_{0}^{a}=x^{a} \frac{W(r, t)}{g r}, \quad W_{\mu}^{a}=\varepsilon_{\mu a b} x^{b} \frac{K(r, t)-1}{g r^{2}}+\left(\delta_{\mu}^{a}-\frac{x^{a} x_{\mu}}{r^{2}}\right) \frac{S(r, t)}{g r}$,

where $\mu=1,2,3$. The example of exact solution for the YM equation (2) in metric (4) has been obtained in [11] with the help of the following substitution

$W=\frac{d \alpha(t)}{d t}, K=G(r) \cos \alpha(t), S=G(r) \sin \alpha(t)$,

where $\alpha(t)$ is an arbitrary function of time.

As a result of simple calculation on the base of (2), (7) and (8), we have the following expressions for the YM tensor components

$\mathbf{F}_{12}=g^{-1}(\mathbf{m} \cos \alpha+\mathbf{l} \sin \alpha) \frac{d G}{d r}, \mathbf{F}_{13}=g^{-1} \sin \theta(\mathbf{m} \sin \alpha-\mathbf{l} \cos \alpha) \frac{d G}{d r}, \mathbf{F}_{23}=g^{-1} \mathbf{n} \sin \theta\left(G^{2}(r)-1\right), \mathbf{F}_{01}=\mathbf{F}_{02}=\mathbf{F}_{03}=0$,

where $\mathbf{n}=(\sin \theta \cos \phi, \sin \theta \sin \phi, \cos \theta), \mathbf{l}=(\cos \theta \cos \phi, \cos \theta \sin \phi,-\sin \theta), \mathbf{m}=(-\sin \phi, \cos \phi, 0)$ are the orthonormalized isoframe vectors. As it is noted in [11], the YM fields (9) have only magnetic components.

Taking into account the YM tensor components (9), one can obtain the following components of EMT (4) for the YM field

$T_{1}^{1}=\frac{1}{8 \pi g^{2} a^{4} \xi^{2}}\left(-2 G^{2}+\frac{\left(G^{2}-1\right)^{2}}{\xi^{2}}\right), \quad T_{2}^{2}=T_{3}^{3}=-\frac{1}{8 \pi g^{2} a^{4}} \frac{\left(G^{2}-1\right)^{2}}{\xi^{4}}, \quad T_{0}^{0}=\frac{1}{8 \pi g^{2} a^{4} \xi^{2}}\left(2 G^{2}+\frac{\left(G^{2}-1\right)^{2}}{\xi^{2}}\right)$,

and $T_{0}^{1}=0$, where the prime means a derivative with respect to $r$

\section{Problem description}

The set of equations for the YM fields (3) can be written down with the help of ansatz (7) as follows

$\left(\xi^{2} W^{\prime}\right)^{\prime}+2[(\dot{S}-W K) K-(\dot{K}+W S) S]=0$

$a \frac{\partial}{\partial t}[a(\dot{S}-W K)]-S^{\prime \prime}+\left[\frac{\left(K^{2}-1+S^{2}\right) S}{\xi^{2}}-(\dot{K}+W S) W\right]=0$

$a \frac{\partial}{\partial t}[a(\dot{K}+W S)]-K^{\prime \prime}+\left[\frac{\left(K^{2}-1+S^{2}\right) K}{\xi^{2}}+(\dot{S}-W K) W\right]=0$.

One can readily verify that the substitution of ansatz (8) into equations (11) yields the only equation for unknown function $G(r)$, namely

$\xi^{2}(r) G^{\prime \prime}-\left(G^{2}-1\right) G=0$.

In our knowledge, the only non-trivial exact solutions for the equation (12) were found by the author (see, for example, [11]) in the following form

$G(r)= \pm \xi^{\prime}(r)=\left\{\begin{array}{cl} \pm \cos (r), & k=+1 \\ \pm 1, & k=0, \\ \pm \cosh (r), & k=-1\end{array}\right.$ 
Considering EMT of the YM gauge field as the EMT of a perfect fluid, that is

$T_{i k}=\left(\rho_{g}+p_{g}\right) u_{i} u_{k}-p_{g} g_{i k}$,

we can get from (10) and (13) that

$T_{0}^{0}=\rho_{g}=\frac{3|k|}{8 \pi g^{2} a^{4}}, T_{1}^{1}=T_{2}^{2}=T_{3}^{3}=-p_{g}=-\frac{|k|}{8 \pi g^{2} a^{4}}$,

Thus, the effective equation of state of the given YM field is the same one as for the pure radiation, that is $p_{g}=\rho_{g} / 3$, when $k= \pm 1$.

As can be seen, the conservation equation for YM field, $T_{i ; k}^{k}=0$, for EMT of YM field

$\dot{\rho}_{g}+3 H\left(\rho_{g}+p_{g}\right)=0$,

where $H=\dot{a} / a$ is the Hubble parameter, is satisfied identically by our solution (15). Simultaneously, the continuity equation in the case of a spatially flat FRW universe, when $k=0$, is nothing more than the equality of $\rho_{g}$ and $p_{g}$ to zero according to (15). That is why we would like to obtain such a solution of equation (12) in this case which could be able to give a non-zero energy $\rho_{g}$, being even an approximate solution. For this purpose, equation (12) in the case of $k=0$ and, consequently, $\xi(r)=r$, that is

$r^{2} G^{\prime \prime}-\left(G^{2}-1\right) G=0$,

can be rewritten for the unknown function $G(x)$ as follows

$\frac{d^{2} G}{d x^{2}}-\frac{d G}{d x}+G-G^{3}=0$,

upon the substitution

$r=\exp (x), \quad x \in(-\infty,+\infty)$.

\section{Solution of the YM equation via Homotopy perturbation method}

In order to briefly recall the Homotopy Perturbation Method, let us consider the following nonlinear differential equation:

$A(u)=f(r), x \in \Omega$,

supplied with boundary conditions $B(u, \partial u / \partial n)=0 ; x \in \Gamma$, where $A$ is a general differential operator, $B$ is a boundary operator, $f(x)$ is a known analytic function, $\Gamma$ is the boundary of the domain $\Omega$. Suppose the operator $A$ can be divided into two parts: $L$ and $N$. Therefore, (20) can be rewritten as follows:

$L(u)+N(u)=f(x)$.

The homotopy $v(x, p): \Omega \times[0,1] \rightarrow \mathbb{R}$ constructed as follows [14]

$H(v, p)=(1-p)\left[L(v)-L\left(v_{0}\right)\right]+p[A(v)-f(x)]=0$,

where $x \in \Omega$ and $p \in[0,1]$ is an embedding parameter, and $v_{0}$ is an initial approximation of (22). Hence, one can see that

$H(v, 0)=L(v)-L\left(v_{0}\right)=0, H(v, 1)=A(v)-f(x)=0$,

and changing the variation of $p$ from 0 to 1 is the same as changing $H(v, p)$ from $L(v)-L\left(v_{0}\right)$ to $A(v)-f(x)$, which are called homotopic. In topology, this is called deformation. Due to the fact that $0 \leq p \leq 1$ can be considered as a small parameter, by applying the perturbation procedure, one can assume that the solution of (22) can be expressed as a series in $p$, as follows:

$v=v_{0}+p v_{1}+p^{2} v_{2}+p^{3} v_{3}+\ldots$

When we put $p \rightarrow 1$, then equation (22) corresponds to (20), and (24) becomes the approximate solution of (20), that is

$u(x)=\lim _{p \rightarrow 1} v=v_{0}+v_{1}+v_{2}+v_{3}+\ldots$

It should be noted that the series (24) is convergent for most cases. However, the convergent rate depends upon the nonlinear operator $A(v)$. Sometimes, even the first approximation is sufficient to obtain the exact solution [13]. As it is emphasized in [14] and [15], the second derivative of $N(v)$ with respect to $v$ must be small, because the parameter $p$ may be relatively large, i.e. $p \rightarrow 1$, and the norm of $L^{-1} \partial N / \partial v$ must be smaller than one, in order that the series converges.

Let us apply the HPM for solving the equation (18). For this end, we suppose the following homotopy

$\frac{d^{2} G}{d x^{2}}-\frac{d G}{d x}+p\left(G-G^{3}\right)=0, p \in[0,1]$

and assume that the solution of (26) can be expressed as a series in $p$ by

$G(x)=G_{0}(x)+p G_{1}(x)+p^{2} G_{2}(x)+\ldots$.

The initial condition $G_{0}(0)$ and $G_{0}^{\prime}(0)$ can be arbitrarily chosen. Here we put

$G_{0}(0)=1+\beta, G_{0}^{\prime}(0)=\beta$, 


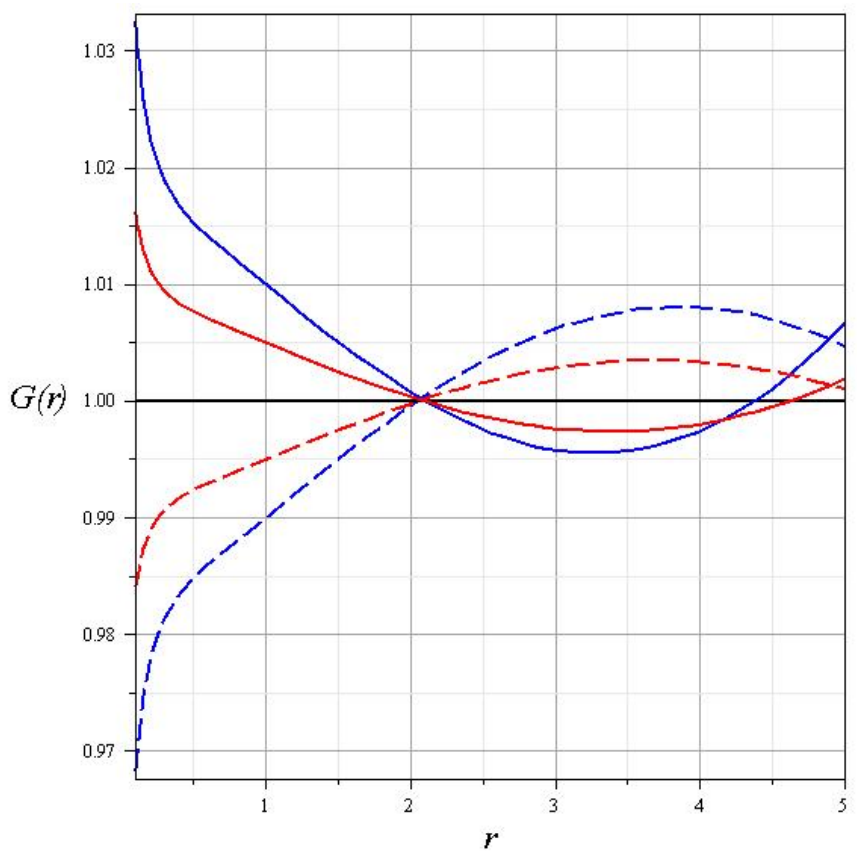

Figure 1: The approximate solution for the radial function $G(r)$ versus $r$, given by Eq. (36), with $\beta=0.01$ (blue solid line), $\beta=-0.01$ (blue dashed line) and $\beta=0.005$ (red solid line), $\beta=-0.005$ (red dashed line).

where $\beta$ is a constant, and

$G_{i}(0)=G_{i}^{\prime}(0)=0$

when $i \geq 1$. Thus, the solution (27) should satisfy the following conditions: $G(0)=1+\beta, G^{\prime}(0)=\beta$. The substitution of (27) into equation (26) yields the following set of linear equations

$p^{0}: G_{0}^{\prime \prime}-G_{0}^{\prime}=0$,

$p^{1}: G_{1}^{\prime \prime}-G_{1}^{\prime}+G_{0}-G_{0}^{3}=0$,

$p^{2}: G_{2}^{\prime \prime}-G_{2}^{\prime}+G_{1}-3 G_{0}^{2} G_{1}=0$

were the prime stands for the derivative with respect to $x$. It is noteworthy that we obtain the infinite set of linear equations. The exact solution for equation (30) with the initial condition given by (28) can be readily obtained as follows

$G_{0}(x)=1+\beta e^{x}$

Substituting this function in equation (31) and taking into account (29), we can get

$G_{1}(x)=\frac{\beta}{6}\left[12+9 \beta+2 \beta^{2}+\left(12 x-12-18 \beta-3 \beta^{2}\right) e^{x}+9 \beta e^{2 x}+\beta^{2} e^{3 x}\right]$.

Then, inserting (33) and (34) in equation (32), we can readily solve it by using the Maple software to obtain the next iteration as follows

$G_{2}(x)=-\frac{\beta}{360}\left[4320+1620 \beta+1440 x+1080 x \beta-640 \beta^{2}+240 x \beta^{2}-405 \beta^{3}-36 \beta^{4}\right.$

$-\left(4320+6480 \beta-2880 x+720 x^{2}+2160 x \beta+1620 \beta^{2}+2880 x \beta^{2}-300 \beta^{3}+720 x \beta^{3}-135 \beta^{4}\right) e^{x}$

$\left.+\left(4860 \beta-2160 x \beta+2160 \beta^{2}-270 \beta^{3}-180 \beta^{4}\right) e^{2 x}+\left(100 \beta^{2}-360 x \beta^{2}+540 \beta^{3}+90 \beta^{4}\right) e^{3 x}-165 \beta^{3} e^{4 x}-9 \beta^{4} e^{5 x}\right]$.

Thus, with the help of (33)-(35), the approximate solution $G \approx G_{0}+G_{1}+G_{2}$ of equation (18) can be obtained as the function of the radial variable $r=\exp (x)$ as follows

$G(r)=1-\beta\left[10+3 \beta+4 \ln r+3 \beta \ln r-\frac{19}{9} \beta^{2}+\frac{2}{3} \beta^{2} \ln r-\frac{9}{8} \beta^{3}-\frac{1}{10} \beta^{4}\right.$

$-\left(11+15 \beta-8 \ln r+2(\ln r)^{2}+6 \beta \ln r+4 \beta^{2}+8 \beta^{2} \ln r-\frac{15}{18} \beta^{3}+2 \beta^{3} \ln r-\frac{3}{8} \beta^{4}\right) r$

$\left.+\beta\left(12-6 \ln r+6 \beta-\frac{3}{4} \beta^{2}-\frac{1}{2} \beta^{3}\right) r^{2}+\beta^{2}\left(\frac{1}{9}-\ln r+\frac{3}{2} \beta+\frac{1}{4} \beta^{2}\right) r^{3}-\frac{33}{72} \beta^{3} r^{4}-\frac{1}{40} \beta^{4} r^{5}\right]$.

For some different numerical values of parameter $\beta$, this function is plotted in Fig. 1. In Fig. 2, we show the instant profile of the EMT components of YM field (10) for $\xi(r)=r$ in the units of $\left(1 / 8 \pi g^{2} a^{4}\right)$. As it is known, the trace of the energy-momentum tensor for a 


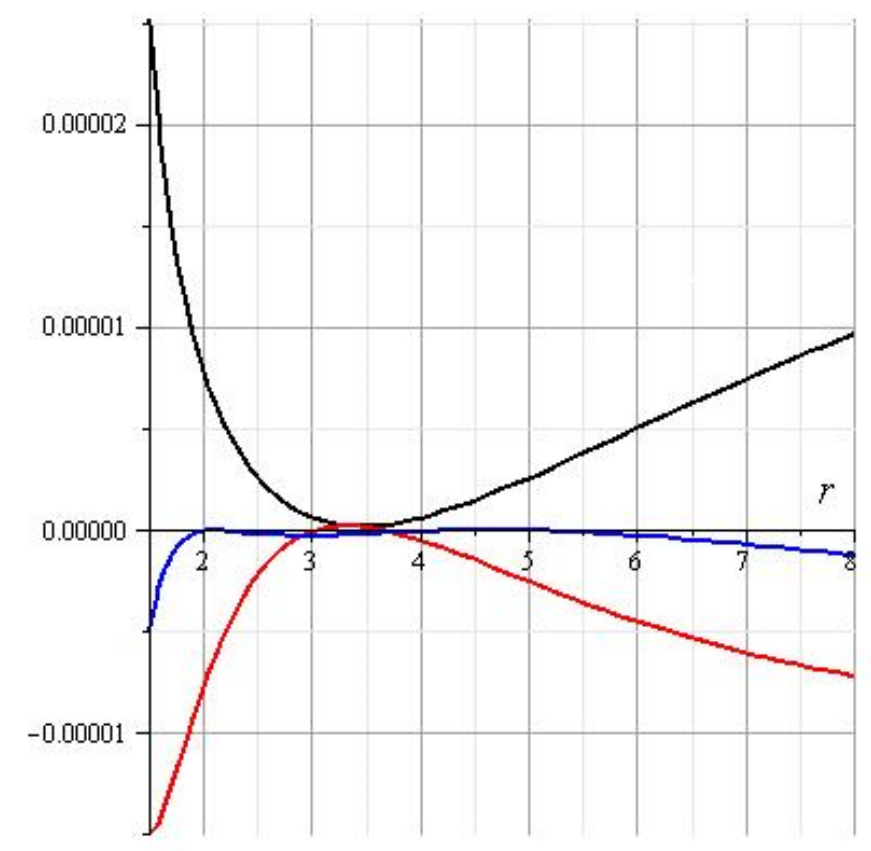

Figure 2: Shows the instant profile of the EMT components (10), namely $T_{0}^{0}$ (in black), $T_{1}^{1}$ (in red), and $T_{2}^{2}=T_{3}^{3}$ (in blue), for the approximate solution (36) in the units of $\left(1 / 8 \pi g^{2} a^{4}\right)$ with $\beta=0.005$.

massless Yang-Mills field (4) is zero, $T_{i}^{i}=0$. It is easy to verify that this equality is identically satisfied by the components of the EMT (10) and, consequently, by the solution (36), that can be seen in Fig. 2. From this figure, it is also seen that the equality $T_{1}^{1}=T_{2}^{2}=T_{3}^{3}$ is not satisfied by the solution (36). But this equality follows from the representation of the energy-momentum tensor of the Yang-Mills field as the EMT of a perfect fluid (14), and it was used in [12] to construct the exact solutions (13) for equation (12). This means that it was the assumption of inequality $T_{1}^{1} \neq T_{2}^{2}=T_{3}^{3}$ that made it possible to obtain a nontrivial solution of the radial equation. Moreover, this result gives a hint about the direction in which one could move in order to obtain some new exact solutions to the radial equation (17).

\section{Conclusion}

Dr. He's Homotopy Perturbation Method has been used in this article for solving the Yang-Mills equation on the gravity background of the flat FRW universe as an efficient method for obtaining the most accurate solution of a highly nonlinear differential equation with an initial condition. For this end, we used the Wu-Yang ansatz that allowed us to reduce the Yang-Mills set of differential equations in the flat FRW universe to a single radial equation for the radial function. As a result of a appropriate replacement of the radial variable, this radial equation was reduced to a form suitable for solving by the HPM. Then, applying HPM, we have found an approximate solution for the components of the Yang-Mills field and graphically illustrate the non-zero character of the components of the energy-momentum tensor of this field on the gravity background of the flat FRW universe. Obviously besides HPM there are other methods for obtaining accurate analytical approximations to nonlinear differential equations which can be used in the problem considered in the present paper.

\section{References}

[1] A. G. Riess, et al. , "Observational Evidence from Supernovae for an Accelerating Universe and a Cosmological Constant", Astronomical Journal, Vol. 116, (1998), 1009. http://dx.doi.org/10.1086/300499

[2] S. Perlmutter, et al., "Measurements of Omega and Lambda from 42 High-Redshift Supernovae", Astrophysical Journal, Vol. 517 (1999), 565. http://dx.doi.org/10.1086/307221

[3] N. Jarosik, C.L. Bennett, et al., "Seven-Year Wilkinson Microwave Anisotropy Probe (WMAP) Observations: Sky Maps, Systematic Errors, and Basic Results", Astrophysical Journal Supplement Series, 192 (2011), 14. http://dx.doi.org/10.1088/0067-0049/192/2/14.

[4] Abdel Nasser Tawfik and Eiman Abou El Dahab3, "Review on Dark Energy Models", Gravitation and Cosmology, 2019, Vol. 25, No. 2, pp. 103-115. http://dx.doi.org/10.1134/S0202289319020154

[5] D. Huterer and D. L. Shafer, "Dark energy two decades after: Observables, probes, consistency tests", Rept. Prog. Phys., 81, 016901 (2018). http://dx.doi.org/10.1088/1361-6633/aa997e

[6] L. Sebastiani, S. Vagnozzi, and R. Myrzakulov, "Mimetic Gravity: A Review of Recent Developments and Applications to Cosmology and Astrophysics", Advances in High Energy Physics, Volume 2017, Article ID 3156915, 43 pages. https://doi.org/10.1155/2017/3156915

[7] C.N. Yang , R.L. Mills , "Conservation of isotopic spin and isotopic gauge invariance", Physical Review, 96, 1 (1954), pp. 191 - 195. http://dx.doi.org/10.1103/PhysRev.96.191

[8] D. V. Gal'tsov, E.A. Davydov, ’Yang-Mills Condensates in Cosmology, International Journal of Modern Physics: Conference Series, 14 (2012) $316-325$. http://dx.doi.org/10.1142/S201019451200743X

[9] V. K. Shchigolev, "Modelling Cosmic Acceleration in Modified Yang-Mills Theory", Gravitation and Cosmology, Vol. 17, No. 3 (2011) 272-275. http://dx.doi.org/10.1134/S0202289311030078

[10] V. K. Shchigolev, G. N. Orekhova, "Non-Minimal Cosmological Model in modified Yang-Mills Theory", Modern Physics Letters A, Vol. 56, No. 2 (2011) 389-396. http://dx.doi.org/10.1142/S0217732311036462

[11] V. K. Shchegolev, K. Samaroo, "Generalized Exact Cosmologies with Interacting Yang-Mills and Nonlinear Scalar Fields", General Relativity and Gravitation, 36(7) (2004), 1661. http://dx.doi.org/10.1023/B:GERG.0000032158.16161.1b

[12] V. K. Shchigolev, D. N. Bezbatko, 'Exact Cosmological Models with Yang - Mills Fields on Lyra Manifold”, Gravit. Cosmol., 24 (2018), 161. https://doi.org/10.1134/S0202289318020135 
[13] J.-H. He, "Homotopy perturbation technique", Computer Methods in Applied Mechanics and Engineering, 178 (1999), 257-262. http://dx.doi.org/10.1016/S0045-7825(99)00018-3

[14] J.-H. He, "A coupling method of homotopy technique and perturbation technique for nonlinear problems", International Journal of Non-Linear Mechanics, 35 (1) (2000), 37-43. http://dx.doi.org/10.1016/S0020-7462(98)00085-7

[15] L. Cveticanin, "Homotopy-perturbation method for pure nonlinear differential equation", Chaos, Solitons \& Fractals, vol. 30, No. 5, 1221 - 1230, 2006. doi:10.1016/j.chaos.2005.08.180

[16] V. Shchigolev, "Homotopy Perturbation Method for Solving a Spatially Flat FRW Cosmological Model", Universal Journal of Applied Mathematics, 2(2) (2014), 99-103. http://dx.doi.org/10.13189/ujam.2014.020204

[17] V. Shchigolev, "Analytical Computation of the Perihelion Precession in General Relativity via the Homotopy Perturbation Method", Universal Journal of Computational Mathematics, 3(4) (2015), 45-49. http://dx.doi.org/10.13189/ujcmj.2015.030401

[18] V. K. Shchigolev, "Calculating Luminosity Distance versus Redshift in FLRW Cosmology via Homotopy Perturbation Method", Gravitation and Cosmology, 23 (2017) 142. http://dx.doi.org/10.1134/S0202289317020098

[19] V. K. Shchigolev, D.N. Bezbatko "Studying Gravitational Deflection of Light by Kiselev Black Hole via Homotopy Perturbation Method", General Relativity and Gravitation, (2019) 51:34. http://dx.doi.org/10.1007/s10714-019-2521-6

[20] Abdul Aziz, Saibal Ray, Farook Rahaman, "A generalized model for compact stars", European Physical Journal C, 76 (2016), 248. http://dx.doi.org/10.1140/epjc/s10052-016-4090-0

[21] F. Rahaman, S. Ray, A. Aziz, S. R. Chowdhury, D. Deb, Exact Radiation Model For Perfect Fluid Under Maximum Entropy Principle, Online available from arXiv:1504.05838 (2015)

[22] A. Aziz, S. Ray, F. Rahaman, M. Khlopov and B. K. Guha, "Constraining values of bag constant for strange star candidates", International Journal of Modern Physics D, http://dx.doi.org/10.1142/S0218271819410062 